Rabaska

Revue d'ethnologie de l'Amérique française

\title{
CoviD-19 et patrimoine au Québec
}

\section{Philippe Dubé}

Volume 18, 2020

URI : https://id.erudit.org/iderudit/1072917ar

DOI : https://doi.org/10.7202/1072917ar

Aller au sommaire du numéro

Éditeur(s)

Société québécoise d'ethnologie

ISSN

1703-7433 (imprimé)

1916-7350 (numérique)

Découvrir la revue

Citer ce document

Dubé, P. (2020). Covid-19 et patrimoine au Québec. Rabaska, 18, 266-270.

https://doi.org/10.7202/1072917ar d'utilisation que vous pouvez consulter en ligne.

https://apropos.erudit.org/fr/usagers/politique-dutilisation/ 


\title{
Opinion
}

\section{Covid-19 et patrimoine au Québec}

\author{
PhilipPe Dubé \\ Professeur retraité de l'Université Laval
}

Il n'est pas ici question de jouer avec les mots, encore moins de faire des amalgames malheureux, mais plutôt d'amener un autre son de cloche à la réflexion faite au sort réservé aux vieux et à l'ancien au Québec. Tous deux semblent loger à la même enseigne : éloignez de ma vue ce qui n'est plus utile, est périmé et, surtout, rappelle une époque révolue. Bien que certains témoignent d'une opinion contraire, je crois que la relation que les Québécois entretiennent avec le passé joue trop souvent à la défaveur du simple respect pour tout ce qui nous a précédé, c'est-à-dire les personnes humaines comme les traces de l'histoire comprises comme patrimoine collectif. Pourtant, notre devise - le fameux « Je me souviens »- est claire à cet égard et devrait, de fait, nous rassembler tous et toutes fébrilement sous un même appel à la mémoire nationale ; mais la réalité semble tout autre. À la vérité, nous assistons publiquement à une sorte d'abandon de ce qui ne compte plus à nos yeux. Faites simplement l'exercice pour savoir qui, dans votre entourage, va au cimetière rendre visite aux morts et vous obtiendrez assez rapidement la réponse $^{1}$. Ce seul fait révèle un rapport au défunt qui en dit long sur le lien que nous entretenons avec le « trépassé » et le «passé », si je puis dire. Ces deux choses relevant d'une relation ambigüe qui devrait nous faire réfléchir. Vœu pieux et réalité ne font pas bon ménage.

Avant la Covid-19, combien y a-t-il eu d'alertes sur l'état de certains CHSLD, décriés à maintes reprises comme insalubres et indignes alors que les pouvoirs publics ont plutôt fait la sourde oreille aux doléances exprimées ?

1. En 2008, Jean Simard, en présentation de son ouvrage Cimetières. Patrimoine pour les vivants (en collaboration avec François Brault, Québec, Éditions GID, 2008, p. 9), signalait déjà le problème : « Nos contemporains ont un rapport ambigu avec le cimetière, rapport fait tout à la fois de répulsion et d'attachement. Le cimetière éloigne parce qu'il nous confronte à l'échéance inéluctable. Par ailleurs il attire du fait qu'il parle des autres, de ceux qui forment désormais l'arrière-garde du groupe : famille, village ou quartier, profession. » 
Tout comme du côté du patrimoine où, de démolitions injustifiées aux destructions sauvages, on a vu disparaître, sous nos yeux impassibles, des monuments qui méritaient pourtant un bien meilleur sort. Nous voici donc aujourd'hui placés devant cette dure réalité alors que nous sommes tous également touchés dans notre propre intégrité sanitaire par une crise pandémique mondiale. Où en sommes-nous au juste collectivement devant tant d'indifférence vis-à-vis le legs de notre passé, qu'il soit humain ou patrimonial ? Serait-t-il vu comme révolu, comme un passé simplement « dépassé »? On doit profiter des circonstances - en ces temps difficiles - pour $\mathrm{y}$ réfléchir de manière transversale, c'est-à-dire en mettant sur la table tous les aspects de la santé d'un peuple, sa mémoire comme ses devoirs envers le mieux-être de tous les citoyens, y compris les disparus.

Comme dans un mauvais film, depuis quelques années nous assistons pantois au spectacle plutôt triste d'une série de destructions préméditées de bâtiments qui sont jugés désuets, obsolètes, nuisibles, voire même dangereux pour la sécurité publique. On produit des dossiers les discréditant pour s'en débarrasser au plus vite, dans le but précis de libérer l'espace public de certains avatars d'un autrefois qu'on ne veut plus voir. Plus encore, on souhaite surtout ne pas en préserver un souvenir pouvant servir à la mémoire d'une communauté ou, plus désastreux encore, celle d'un peuple tout entier. Ce ne sont pas les autres - des étrangers, comme on dit - qui agissent de la sorte pour nous signifier brutalement de débarrasser le plancher, mais bel et bien nous-mêmes qui en prenons l'initiative pour faire preuve malhabilement d'une soi-disant bonne et saine administration des fonds publics.

Signalons, au passage, quelques exemples qui se sont multipliés ces derniers temps et qui illustrent le processus de déchéance patrimoniale au même titre qu'on observe la mise à l'écart de la société active des personnes âgées jugées, par leur fragile état de santé, comme préjudiciables au bon équilibre de la collectivité. Avant qu'elles ne rendent l'âme, on préfère les parquer dans des immeubles qui les confineront à l'oubli le plus rapidement possible et ainsi les retireront définitivement du paysage de nos vies affairées. Par une courte revue, on évoquera ici des cas, reconnus comme des « petits patrimoines » ou encore du " patrimoine de proximité », pour souligner avec encore plus d'acuité à quel point même la mémoire des petites choses nous laisse collectivement indifférents. Pourtant, cette mémoire du quotidien, du banal, est peut-être la plus simple à conserver parce qu'elle ne représente pas d'enjeux symboliques majeurs, sauf pour la communauté qui la soutient. L'intérêt pécuniaire de certains promoteurs immobiliers ou l'avidité de municipalités pour quelques deniers supplémentaires y font parfois une différence. C'est d'ailleurs pour ces mêmes raisons de rationalisation 
financière qu'on laisse aller à vau-l'eau nos vieillards qui glissent sans bruit dans la honte d'eux-mêmes vers une mort imminente et humiliante.

\section{Démolitions, abandons et mobilisations}

Côté patrimonial, un rapide tour de piste nous permet de constater que les pertes encourues du point de vue de l'héritage historique sont aussi palpables que le malaise de plus en plus perceptible qu'elles suscitent dans la population, le plus souvent locale. Pensons simplement à la maison Busteed de la Pointeà-la-Croix dans la Baie des Chaleurs en Gaspésie pour mesurer l'ampleur du mal. Construite vers 1800 et jalousement préservée par les descendants de Thomas Busteed jusqu'en 2009, elle était reconnue comme la plus ancienne construction de la région gaspésienne pour finalement disparaître en une seule nuit, celle du 31 mai au $1^{\text {er }}$ juin 2020, en proie aux flammes d'un incendie probablement d'origine criminelle. Plus de 200 ans d'histoire, solide et matérielle, sont réduits en cendres et partent en fumée comme par enchantement, comme s'il existait moult témoins d'une période aussi profonde qui ont marqué au fer rouge la péninsule. Patrimoine revendiqué ou pas, il est ici l'objet d'une mémoire refoulée que l'on semble vouloir oublier bien que l'histoire ne puisse être évacuée, même par le feu. Les blessures anciennes sont là pour rester. Nous, contemporains, sommes là pour les soigner, sinon les guérir en leur donnant un sens. Que dire maintenant de la maison Pasquier à Québec, une autre habitation privée - tricentenaire cette fois - que la famille Paquet a occupé pendant huit générations consécutives depuis 1698 et qui a finalement été rasée à la fin septembre 2019 parce que la Ville de Québec ne lui reconnaissait pas suffisamment de caractère patrimonial pour empêcher sa démolition. Le 15 mai dernier, à Saint-Joseph en Beauce, même scénario pour la maison du XVIII ${ }^{\mathrm{e}}$ siècle où avait séjourné le général Benedict Arnold à l'automne 1775 avant de tenter d'attaquer Québec avec ses troupes. Un fait historique qui remonte à 245 ans. Encore une fois, une démolition approuvée par les autorités municipales. On doit tout de même être saisi du fait que la population locale de l'époque appuyait ouvertement la mission du général étatsunien qui souhaitait par sa ruse militaire ébranler les fondations naissantes de l'Empire britannique en Amérique du Nord. La perte est ici totale et, du coup, nous prive d'une page importante d'histoire et d'archéologie qui aurait pu nous en apprendre davantage sur les tenants et aboutissants de la guerre d'indépendance des États-Unis dont la naissance nationale date de 1776.

$\mathrm{Au}$ chapitre des négligences et des laissés-pour-compte, il y a, en Charlevoix, la triste destinée de la maison du peintre René Richard, située sur le domaine Cimon que la famille habite depuis 1881, mise en vente au plus offrant et laissée à l'abandon dans un état lamentable depuis 2018 . Quelle perte sèche pour le patrimoine régional et national alors que le peintre 
est le maître incontesté du paysage brut et sauvage, et que sa présence a marqué à tout jamais Baie-Saint-Paul. Récemment, on signale que le long de la route du Mitan qui traverse l'Île d'Orléans du nord au sud, reliant les villages de Sainte-Famille et de Saint-Jean, on a planté sans permis, et sur quelques kilomètres, une série de poteaux électriques enfilant ses câbles aériens pour les besoins d'une cabane à sucre privée. Évidemment, il s'agit là d'une offense grave infligée de plein fouet au paysage patrimonial de l'Île qui possède, comme on le sait, le statut de site patrimonial en vertu de la Loi sur le patrimoine culturel. Tout ceci crée, il va sans dire, un réel émoi dans la population locale comme ce fut aussi le cas lors de la destruction de la très élégante corniche, avec sa console et ses parapets, du café-bar les Quatre Jeudis à Gatineau où une véritable prise de bec a impliqué la mairie, un groupe de citoyens et le propriétaire de l'immeuble. L'esclandre public laissera évidemment une plaie dans la population qui se sent foncièrement lésée par la perte de cette marque architecturale du quartier patrimonial du Hull ancien. Depuis ce drame, la mairie semble mieux disposée à revoir ses pratiques et à resserrer les règles en matière de patrimoine. Pour rester dans l'Outaouais, on vient d'apprendre tout récemment le rasage autorisé, et sans avis public, du petit temple presbytérien St. Andrew à Cantley, construit en 1877. Localement, on dénonce ouvertement ce geste absurde en invoquant le fait que la pandémie de la Covid-19, qui occupe toute l'attention médiatique, laisse en quelque sorte le champ libre aux autorités qui agissent sans consulter la population, ni débat public.

Dans ce qui peut ressembler, à bien des égards, à une véritable hécatombe, il y aussi des histoires plus heureuses pour la mémoire collective, celles qui connaissent justement des revirements de situation spectaculaires et qui donnent encore un peu d'espoir. En effet, quand on observe des actions de mobilisation spontanée ici et là sur le territoire, on doit après tout s'en réjouir. C'est ce qui se passe notamment à Québec avec l'église du TrèsSaint-Sacrement, condamnée d'emblée par le diocèse, et que la seule action d'un regroupement de citoyens du quartier a permis de renverser la vapeur et d'amener le dossier sur le terrain d'autres solutions. On se dit alors que tout n'est pas perdu. De même, ici à La Pocatière, alors que la meunerie et la bergerie de la ferme-école Lapotika étaient toutes deux jugées inaptes à prodiguer leurs services pour cause de désuétude ; le vent vient brusquement de tourner après un appel à la mobilisation populaire pour empêcher cette opération d'éradication. Le permis de démolition a donc été suspendu afin de permettre au ministère de la Culture d'accompagner les propriétaires, deux partenaires gouvernementaux, le MAPAQ et la SQI, dans une démarche de requalification des lieux pour éviter la solution de facilité, celle de détruire pour mieux reconstruire. 


\section{Émotions patrimoniales}

Quand on y regarde de près, le dénominateur commun que partage ces malheureux cas de destruction ou de menace de disparition de notre patrimoine - et combien d'autres encore - est sans contredit la part d'affect qu'ils emportent avec eux. Vu sous cet angle et au-delà des circonstances, on est à même de constater qu'il y a effectivement apparition d'une nouvelle sensibilité au passé qui se manifeste par l'éclosion de regroupements spontanés autour de bâtiments ou de sites en perdition. Ici on dénonce parce qu'on a " altéré l'âme du lieu », là-bas on reconnaît plutôt « avoir perdu une partie de nous-mêmes ». Comme le démontre l'ethnologue Daniel Fabre², le patrimoine devient de plus en plus pluriel. Il est aujourd'hui moins le résultat d'une expertise proclamée en haut lieu que d'une appropriation collective qui nous ramène sur le plateau d'un " régime expérientiel du passé ». Une " histoire d'en bas » commence à s'articuler d'elle-même puisque, par définition, elle échappe à l'emprise des savants pour finalement s'ancrer dans un large public qui se laisse de plus en plus contaminer par les fièvres patrimoniales. Nous pourrions conclure, comme le fait Bérénice Waty dans un article intitulé « Quand le patrimoine devient l'affaire de tous » : « Les émotions patrimoniales attestent d'une volonté des individus de prendre part aux décisions qui concernent le patrimoine mais plus largement leur cadre de vie et les objets de leur quotidien ou de leur histoire (passée, présente ou à venir) : en ce sens, même si l'on parle de lutte ou de conflit, elles sont un témoignage d'une démocratie participative en actions. ${ }^{3}$ "

On comprend ici que la part d'affect largement partagée devient l'ingrédient déclencheur d'une conscience patrimoniale populaire parce qu'elle fait écho et traduit en mots simples l'attachement au passé à travers des objets du quotidien. L'affect et l'intellect doivent nécessairement se conjuguer, mais les émotions deviennent le véhicule qui transporte la cognition. À mon avis, c'est à cette condition que le patrimoine deviendra l'affaire de tous. Si on ne jugule pas la crise patrimoniale ou sanitaire au plus tôt, elle laissera dans le deuil les générations futures qui auront perdu de précieux témoins pour retracer, à petits pas, leur passé. Arrêtons ce carnage en prenant soin de cette mémoire vivante qui nous est léguée à travers les personnes et les empreintes qu'elles laissent. Après tout, a-t-on besoin de rappeler qu'une fois perdue, elle ne revient plus.

2. Daniel Fabre (dir.), Émotions patrimoniales, textes réunis par Annick Arnaud, Paris, Éditions de la Maison des sciences de l'homme, «Ethnologie de la France », cahier n 27, 2013.

3. Bérénice Waty, «Émotions patrimoniales », dans Bulletin des bibliothèques de France, 2016, $n^{\circ} 7$, p. 8-22 (consulté en ligne : bbf.enssib.fr/consulter/bbf-2016-07-0008-001). 